\title{
Data-driven Correction of Models for Deformable Solids
}

\author{
D. González ${ }^{1, b)}$, F. Chinesta ${ }^{2}$ and E. Cueto ${ }^{1, a), c)}$ \\ ${ }^{1}$ Aragon Institute of Engineering Research. Universidad de Zaragoza. Maria de Luna, s. n., E-50018 Zaragoza, \\ Spain. \\ ${ }^{2}$ PIMM Lab and ESI Group. ENSAM ParisTech. 151, Bvd. de l'Hôpital. F-75013 Paris, France. \\ ${ }^{a)}$ Corresponding author: ecueto@unizar.es \\ b)URL: http://amb.unizar.es/gonzal.html \\ c)URL: http://amb.unizar.es/ecueto.html
}

\begin{abstract}
Unveiling physical laws from data is seen as the ultimate sign of human intelligence. While there is a growing interest in this sense around the machine learning community, some recent works have attempted to simply substitute physical laws by data. We believe that getting rid of centuries of scientific knowledge is simply nonsense. There are models whose validity and usefulness is out of any doubt, so try to substitute them by data seems to be a waste of knowledge. While it is true that fitting well-known physical laws to experimental data is sometimes a painful process, a good theory continues to be practical and provide useful insights to interpret the phenomena taking place. That is why we present here a method to construct, based on data, automatic corrections to existing models. Emphasis is put in the correct thermodynamic character of these corrections, so as to avoid violations of first principles such as the laws of thermodynamics. These corrections are sought under the umbrella of the GENERIC framework [M. Grmela and H. Ch. Oettinger, Dynamics and thermodynamics of complex fluids. I. Development of a general formalism. Phys. Rev. E 56, 6620, 1997], a generalization of Hamiltonian mechanics to non-equilibrium thermodynamics. This framework ensures the satisfaction of the first and second laws of thermodynamics, while providing a very appealing context for the proposed automated correction of existing laws. In this work we focus on solid mechanics, particularly large strain (visco-) hyperelasticity.
\end{abstract}

\section{INTRODUCTION}

Teaching machines to learn physical models is undoubtedly a very complex task, yet in recent times many works have bene devoted to this end, e.g., [1, 2, 3, 4, 5, 6]. In this work we attempt a different approach. Instead of starting from scratch, we propose to leverage as much as possible knowledge from well-established constitutive laws, but try to learn corrections from data, where these provide unsatisfactory results.

For this to be possible, we rely on the most general framework that, to our knowledge, allows for a proper satisfaction of the laws of thermodynamics. This framework has been coined as General Equation for Non-Equilibrium Reversible-Irreversible Coupling (GENERIC) [7, 8]. In what follows, we put the existing models into the GENERIC framework and, based on data, learn corrections to these where necessary.

\section{A review of the GENERIC formalism}

\section{The basics}

GENERIC was developed by [7] trying to develop a common structure for non-Newtonian fluid models. Selecting appropriate state variables is the first step in this route and is by no means straightforward in a general case for which no information is available. For most systems - and specially when we start from known models, as it is the case in this work - simple rules exist for the selection of such variables [8].

Let us call these variables $\mathbf{z}_{t}=\mathbf{z}(t): \mathcal{I} \rightarrow \mathcal{S}, \mathbf{z} \in C^{1}(0, T]$, evolving in the interval $\mathcal{I}=(0, T]$. $\mathcal{S}$. Here, $\mathcal{S}$ represents the space in which these variables live, and is therefore problem-dependent. GENERIC aims to establish an expression for the time evolution of these variables, $\dot{\mathbf{z}}(t)$. 
The GENERIC equation reads

$$
\dot{\mathbf{z}}_{t}=\underbrace{\mathbf{L}\left(\mathbf{z}_{t}\right) \nabla E\left(\mathbf{z}_{t}\right)}_{\text {Hamiltonian }}+\underbrace{\mathbf{M}\left(\mathbf{z}_{t}\right) \nabla S\left(\mathbf{z}_{t}\right)}_{\text {Dissipative }}, \quad \mathbf{z}(0)=\mathbf{z}_{0} .
$$

The first sum on the right-hand side term represent the Hamiltonian, or conservative, part of the behavior of the system. The term $\mathbf{L}\left(\mathbf{z}_{t}\right)$ is the Poisson matrix. The second sum takes into account the dissipative behavior of the system, being $\mathbf{M}\left(\mathbf{z}_{t}\right)$ the so-called friction matrix. In turn, $E\left(\mathbf{z}_{t}\right)$ represents the total energy of the system, while $S\left(\mathbf{z}_{t}\right)$ represents its entropy.

For Equation (1) to satisfy basic thermodynamic principles, it must be supplemented with the following degeneracy conditions:

$$
\begin{aligned}
\mathbf{L}(\mathbf{z}) \cdot \nabla S(\mathbf{z})=\mathbf{0}, \\
\mathbf{M}(\mathbf{z}) \cdot \nabla E(\mathbf{z})=\mathbf{0} .
\end{aligned}
$$

This is equivalent to $\mathbf{L}(\mathbf{z})$ being skew-symmetric and $\mathbf{M}$ being symmetric, positive semi-definite. If these conditions are met, then,

$$
\dot{E}(\mathbf{z})=\nabla E(\mathbf{z}) \cdot \dot{\mathbf{z}}=\nabla E(\mathbf{z}) \cdot \mathbf{L}(\mathbf{z}) \nabla E(\mathbf{z})+\nabla E(\mathbf{z}) \cdot \mathbf{M}(\mathbf{z}) \nabla S(\mathbf{z})=0,
$$

which is, in fact, the equation of conservation of energy for the system. Equivalently,

$$
\dot{S}(\mathbf{z})=\nabla S(\mathbf{z}) \cdot \dot{\mathbf{z}}=\nabla S(\mathbf{z}) \cdot \mathbf{L}(\mathbf{z}) \nabla E(\mathbf{z})+\nabla S(\mathbf{z}) \cdot \mathbf{M}(\mathbf{z}) \nabla S(\mathbf{z}) \geq 0,
$$

i.e., the fulfillment of the second principle of thermodynamics.

\section{Correcting models in a GENERIC framework}

The vast majority of hyperelastic models can be straightforwardly cast into the GENERIC structure [9], [10] [11]. Purely hyperelastic materials are strictly conservative, and thus Hamiltonian. Should the material have a dissipative behavior, however, the model needs to be complemented with a dissipative part described by $\mathbf{M}(\mathbf{z})$ and $S(\mathbf{z})$.

Assuming that an inexact model exists, but that it provides unsatisfactory results at least in a part of the experimental campaign, we look for a correction of the form

$$
\mathbf{z}^{\text {corr }}=\mathbf{z}^{\exp }-\mathbf{z}^{\bmod }
$$

where "corr", "exp" and "mod" stand, respectively, for correction, experimental and model. The sought correction in the GENERIC framework will be such that the corrected model will also have a GENERIC structure. This correction will need to be of the form

$$
\dot{\mathbf{z}}^{\text {corr }}=\mathbf{L} \nabla E\left(\mathbf{z}^{\text {corr }}\right)+\mathbf{M} \nabla S\left(\mathbf{z}^{\text {corr }}\right) .
$$

We do not consider a correction for $\mathbf{L}$ nor $\mathbf{M}$, since $\mathbf{L}$ is assumed to be identical to that of the model (we consider the same state variables). We discard any possible $\mathbf{M}$ coming from the inexact model and instead re-compute it from scratch. The resulting, corrected model will be of the form

$$
\dot{\mathbf{z}}^{\exp }=\dot{\mathbf{z}}^{\bmod }+\dot{\mathbf{z}}^{\text {corr }}=\mathbf{L} \nabla E\left(\mathbf{z}^{\text {corr }}\right)+\mathbf{M} \nabla S\left(\mathbf{z}^{\operatorname{corr}}\right)+\mathbf{L} \nabla E\left(\mathbf{z}^{\bmod }\right),
$$

and therefore

$$
\dot{\mathbf{z}}^{\exp }=\mathbf{L}\left(\nabla E\left(\mathbf{z}^{\text {corr }}\right)+\nabla E\left(\mathbf{z}^{\bmod }\right)\right)+\mathbf{M} \nabla S\left(\mathbf{z}^{\text {corr }}\right) .
$$

In other words, the corrected model for $\mathbf{z}^{\mathrm{exp}}$ possesses a GENERIC structure with a correction in the Hamiltonian term.

The proposed method consist therefore in a regression for the terms of the GENERIC model, constrained by the degeneracy conditions, within a time interval $\mathcal{J} \subseteq \mathcal{I}$ :

$$
\mu^{*}=\{\mathrm{M}, \mathrm{DE}, \mathrm{DS}\}=\arg \min _{\mu}\left\|\mathbf{z}(\mu)-\mathbf{z}^{\text {meas }}\right\|,
$$

with $\mathbf{z}^{\text {meas }} \subseteq Z$, a subset of the total available experimental results. DE and DS represent the discrete gradients (in a finite element sense). 


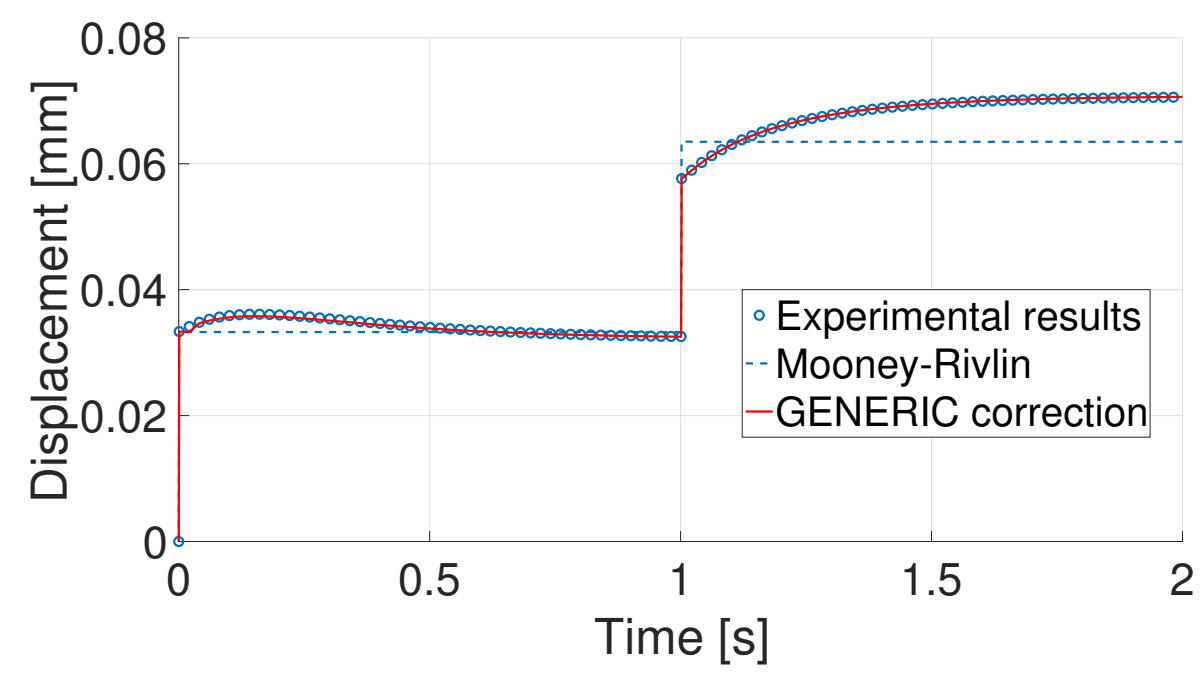

FIGURE 1. Comparison of the Mooney-Rivlin model prediction and its subsequent GENERIC correction with the experimental results for one particular experiment.

\section{Corrections to hyperelastic models}

Pseudo-experimental data have been obtained by finite element simulation of a visco-hyperelastic Mooney-Rivlin material in which

$$
W=C_{1}\left(\bar{I}_{1}-3\right)+C_{2}\left(\bar{I}_{2}-3\right)+D_{1}(J-1)^{2}
$$

with $\bar{I}_{1}=J^{-\frac{2}{3}} I_{1}$ and $\bar{I}_{2}=J^{-\frac{4}{3}} I_{2}$, and where the invariants of the right Cauchy-Green tensor $\mathbf{C}$ are defined as $I_{1}=$ $\lambda_{1}^{2}+\lambda_{2}^{2}+\lambda_{3}^{2}$, and $I_{2}=\lambda_{1}^{2} \lambda_{2}^{2}+\lambda_{2}^{2} \lambda_{3}^{2}+\lambda_{3}^{2} \lambda_{1}^{2}$, respectively. $J$ represents, as usual, the determinant of the gradient of deformation tensor. In this case, $C_{1}=27.56 \mathrm{MPa}, C_{2}=6.89 \mathrm{MPa}$ and $D_{1}=0.0029 \mathrm{MPa}$.

The viscoelastic behavior of this material is assumed to be governed by the shear modulus $G$ and bulk modulus $K$, whose time evolution is modeled by means of a Prony series in terms of the instantaneous moduli,

$$
\begin{aligned}
& \frac{G(t)}{G_{0}}=1-\sum_{i=1}^{2} \bar{g}_{i}^{P}\left(1-\exp \left(-\frac{t}{\tau_{i}}\right)\right), \\
& \frac{K(t)}{K_{0}}=1-\sum_{i=1}^{2} \bar{k}_{i}^{P}\left(1-\exp \left(-\frac{t}{\tau_{i}}\right)\right),
\end{aligned}
$$

with $\bar{g}_{i}^{P}=[0.2,0.1]$ and $\bar{k}_{i}^{P}=[0.5,0.2]$. The relaxation times take the values $\tau_{i}=[0.1,0.2]$ seconds, respectively. With these values, the initial instantaneous Young's modulus takes the value $E=206.7 \mathrm{MPa}$, with Poisson's ratio $v=0.45$.

We simulated 557 different loading processes, subjected to a load history of different amplitudes. In every case, a first plane stress state $\left(\sigma_{x}, \sigma_{y}, \tau_{x y}\right)$-values are not correlated-is applied during a short impulse of 0.021 seconds, then maintained at constant value for one more second, allowing the material to creep. This is followed by a second loading process of 0.021 seconds at a different $\left(\sigma_{x}, \sigma_{y}, \tau_{x y}\right)$ value, followed by a final plateau of one more second. For each one of the 557 different experiments these two stress states were different. These results are stored in the form of 557 different $Z$ vectors, thus representing a trajectory in time.

In Figure 1 results are shown for one of the 557 essays. Experimental results, Mooney-Rivlin prediction and the subsequent GENERIC correction are shown. Experimental results are accurately reproduced after the sought correction is applied. The mean squared error for this particular case (any of the tested trajectories in time provide similar results) was $0.018 \%$. 


\section{Discussion}

A method has been proposed to learn correction to constitutive models from data. These are developed under the framework of GENERIC, so as to satisfy basic principles of thermodynamics. In the results that will be shown, deliberately wrong models are corrected to account for the right dissipative character of the material, providing very accurate results.

\section{ACKNOWLEDGMENTS}

This work has been supported by the Spanish Ministry of Economy and Competitiveness through Grants number DPI2017-85139-C2-1-R and by the Regional Government of Aragon and the European Social Fund, research group T24 17R.

\section{REFERENCES}

[1] S. L. Brunton, J. L. Proctor, and J. N. Kutz, Proceedings of the National Academy of Sciences (2016), 10.1073/pnas.1517384113, http://www.pnas.org/content/early/2016/03/23/1517384113.full.pdf .

[2] B. Peherstorfer and K. Willcox, Computer Methods in Applied Mechanics and Engineering 306, 196 - 215 (2016).

[3] B. Peherstorfer and K. Willcox, Computer Methods in Applied Mechanics and Engineering 291, 21 - 41 (2015).

[4] R. Swischuk, L. Mainini, B. Peherstorfer, and K. Willcox, Computers \& Fluids (2018).

[5] M. Raissi, P. Perdikaris, and G. E. Karniadakis, ArXiv preprint arXiv:1711.10561 (2017).

[6] M. Raissi, P. Perdikaris, and G. E. Karniadakis, ArXiv preprint arXiv:1711.10566 (2017).

[7] M. Grmela and H. C. Öttinger, Phys. Rev. E 56, 6620-6632Dec (1997).

[8] H. C. Öttinger, Beyond Equilibrium Thermodynamics (Wiley, 2005).

[9] L. R. G. Treloar, The physics of rubber elasticity (Clarendon Press, 1975).

[10] R. W. Ogden, Non-linear elastic deformations (Wiley, 1984).

[11] G. A. Holzapfel and T. C. Gasser, Journal of Elasticity 61, 1-48 (2000).

[12] J. Peña, M. Martínez, and E. Peña, Acta Mechanica 217, 63-74 (2011).

[13] A. García, M. A. Martínez, and E. Peña, Biorheology 49, 271-288 (2012). 